\title{
Active gating as a method to inhibit the crosstalk of Single Photon Avalanche Diodes in a shared well
}

\author{
Anna Vilà ${ }^{1}$ Eva Vilella, Andreu Montiel, Oscar Alonso, Angel Dieguez \\ Electronics Department, Physics Faculty, University of Barcelona, C/Martí i Franqués 1, 08028- \\ Barcelona, Spain. avila@el.ub.edu
}

\begin{abstract}
Advances in SPAD arrays propose improving the fill factor by confining several SPADs in the same well, with a main issue related to crosstalk. For measurements triggered only in well-defined time periods that can be known in advance, the pixels can be inhibited before the arrival of the crosstalk charge. This paper reports the crosstalk characterization of in an array of SPADs fabricated in a conventional CMOS technology in the same n-well (fill factor 67\%). A long gating time gives a crosstalk not less than $2.75 \%$, while reducing it below 2.5 ns completely eliminates crosstalk, as predicted by the theory and by TCAD simulations.
\end{abstract}

Keywords: SPAD, APD, avalanche, crosstalk, gating, noise, CMOS.

\section{INTRODUCTION}

So far, Charge Coupled Devices (CCDs) have been the preferred image sensors for a huge range of research and commercial applications, while Monolithic Active Pixel Sensors (MAPSs) provide faster response and easier integration with satellite electronics, Depleted Field Effect Transistors (DEPFETs) provide large signals owing to their internal amplification, and Silicon PhotoMultipliers (SiPMs) are superior in terms of sensitivity, timing response and gain. Still, Single Photon Avalanche Detectors (SPADs) include all these advantages together with high spatial resolution thanks to a readout circuit for every sensor, providing highly-sensitive devices with high speed. The further integration of SPAD sensors and their front-end electronics on a single CMOS die opened the way for commercial applications, including 2D and $3 \mathrm{D}$ vision systems ${ }^{1}$, fluorescence lifetime imaging microscopy ${ }^{2}$, positron emission tomography ${ }^{3}$ and high-energy physics experiments ${ }^{4}$.

Recent advances in SPAD arrays have been proposed to improve the fill factor, by confining several SPADs in the same macropixel sharing their well $1^{5,6}$ and featuring optical fill factors up to $70 \%$. The main issue of this approximation relates to the ease of crosstalk, as spurious uncorrelated avalanches may be triggered in neighboring pixels by the ignition of an avalanche. This crosstalk grows with the signal, and consequently can represent a non-negligible noise in the receiver pixel. In significant applications of SPADs in which the measurement is triggered in specific short and well-known time periods, the detector pixels can be inhibited before the arrival of this spurious charge. This gated-mode operation is expected to limit the absolute noise in each detection cycle, improving the signal-to-noise ratio and the dynamic range of the detector.

To evaluate the effectiveness of this approximation, this paper reports the crosstalk characterization in an array of five SPADs fabricated in a conventional CMOS technology and that share the same n-well to increase fill factor. It reports on the improvements achieved by active gating to inhibit the electrical crosstalk by controlling its gated operation by observation and dead times.

\footnotetext{
1 annavila@ub.edu, phone +34 93 4039170, fax +34 93 4021148, www.ub.edu
}

Photonic Fiber and Crystal Devices: Advances in Materials and Innovations in Device Applications VII, edited by Shizhuo Yin, Ruyan Guo, Proc. of SPIE Vol. 8847, 88470S - (c) 2013 SPIE

CCC code: $0277-786 X / 13 / \$ 18 \cdot$ doi: $10.1117 / 12.2024212$ 


\section{EXPERIMENTAL}

Five avalanche photodiodes $(20 \mu \mathrm{m} \times 100 \mu \mathrm{m})$ were fabricated with the standard HV-AMS $0.35 \mu \mathrm{m}$ CMOS technology. They were parallel in a row (from pixel 0 to 4 ) sharing the same $n$-well to give a fill factor of $67 \%$ (Fig. 1), and consisted in pn junctions reverse biased $\mathrm{V}_{\mathrm{OV}} \mathrm{V}$ above their breakdown voltage $\left(\mathrm{V}_{\mathrm{BD}}\right)$ to operate in the so-called Geiger mode.

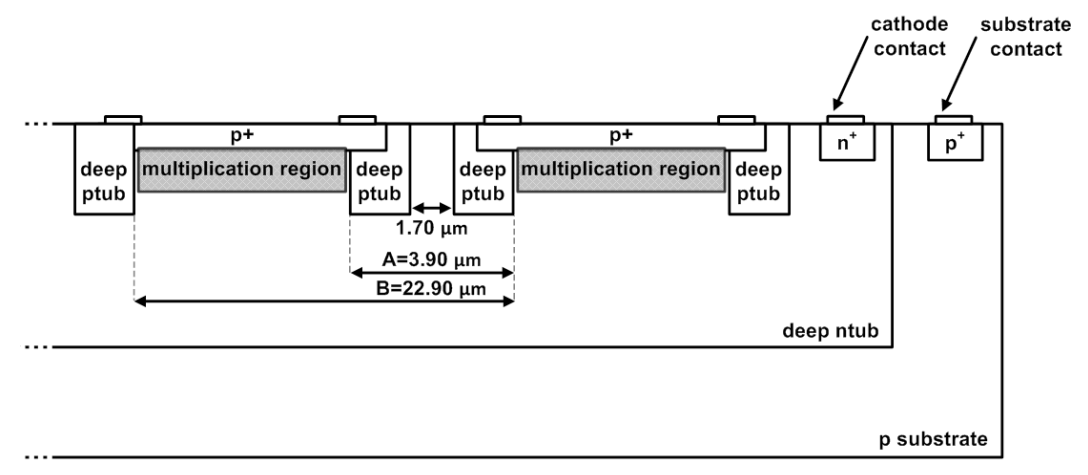

Figure 1. Cross section of a row of the SPAD array fabricated with the standard HV-AMS 0.35 um CMOS technology, showing the distances between neighbouring pixels.

In these conditions, a detected photon produces an electron-hole pair that is accelerated by the strong electric field at the junction. This generates a self-sustained avalanche process resulting in a macroscopic current that needs to be stopped to avoid damaging the device. Passive or active quenching circuits perform this stop operation by lowering the reverse bias voltage down to or below $\mathrm{V}_{\mathrm{BD}}$. Finally, the bias of the sensor needs to be restored to its initial value, to make the sensor sensitive again for upcoming avalanches. Figure 2 shows the schematics of the complete pixel detector, with the photodiode, active inhibition $\left(\mathrm{M}_{\mathrm{P} 0}\right)$ and active reset $\left(\mathrm{M}_{\mathrm{N} 0}\right)$ switches to perform the gated operation, and a readout circuit based on a CMOS inverter $\left(\mathrm{M}_{\mathrm{P} 1}-\mathrm{M}_{\mathrm{N} 1}\right)$, a 1-bit memory register $\left(\mathrm{M}_{\mathrm{N} 2}-\mathrm{M}_{\mathrm{P} 2}-\mathrm{M}_{\mathrm{N} 3}\right)$ and a pass gate $\left(\mathrm{M}_{\mathrm{N} 4}\right)$. In this scheme, it can be considered that the pixels work with passive quenching and active recharge.

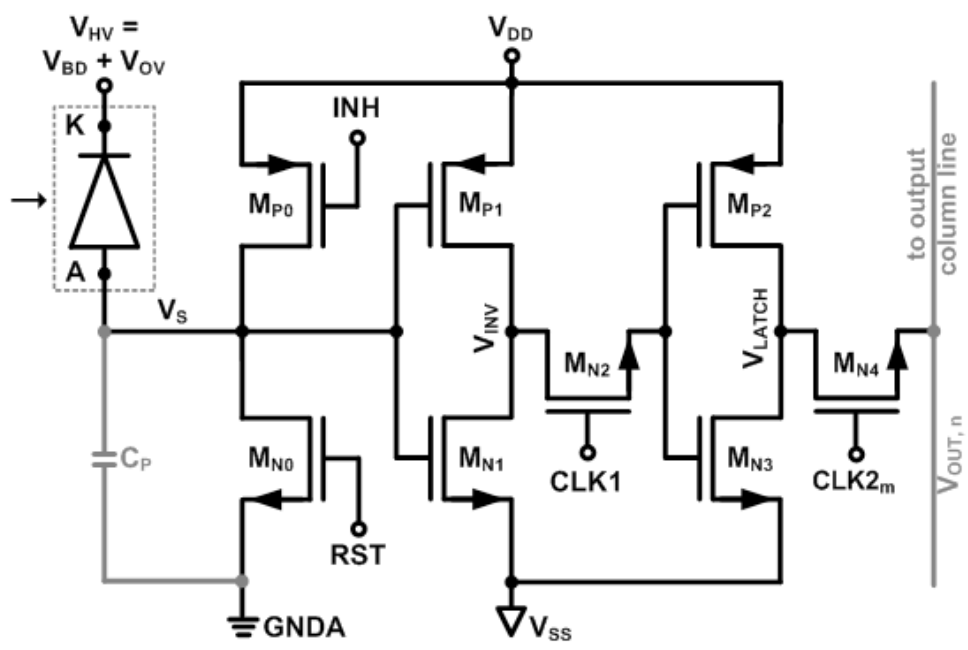

Figure 2. Schematics of the proposed pixel detector showing the photodiode, active inhibition $\left(\mathrm{M}_{\mathrm{P} 0}\right)$ and reset $\left(\mathrm{M}_{\mathrm{N} 0}\right)$ switches, and readout circuit based on a CMOS inverter $\left(\mathrm{M}_{\mathrm{P} 1}-\mathrm{M}_{\mathrm{N} 1}\right)$, a 1-bit memory register $\left(\mathrm{M}_{\mathrm{N} 2}-\mathrm{M}_{\mathrm{P} 2}-\mathrm{M}_{\mathrm{N} 3}\right)$ and a pass gate $\left(\mathrm{M}_{\mathrm{N} 4}\right)$.

A development board based on an ALTERA Cyclone IV FPGA was used to generate the fast logic control signals (RST, INH, CLK1 and CLK2) and also to count off-chip the pulses generated by the sensors. A dedicated software was developed to obtain real-time images of the measured data in a computer. 


\section{RESULTS AND DISCUSSION}

It is known that, when an avalanche occurs in an SPAD, a large quantity of electrons and holes are generated in a very limited volume in the multiplication region. These carriers start to diffuse very quickly in all directions and, according to ISE-TCAD simulations ${ }^{7}$, more than $10^{13}$ holes $/ \mathrm{cm}^{3}$ arrive to the neutral zone just $1 \mathrm{~ns}$ after the avalanche itself, in the case of SPAD sensors like the ones analyzed here. In this region holes are minoritary and start to recombine, but diffusion is still so important that dominates the holes movement in such a way that some of them can reach the neighboring multiplication region, drift towards the surface and generate a new avalanche ascribable to crosstalk. This phenomenon could be described, as a first approximation, by the Fick's diffusion laws. The solution then should be a Gaussian depending on the surface carrier concentration at the limit of the neutral zone (the product between the volume concentration and the depth of the charge zone, what gives $2 \cdot 10^{9} \mathrm{~cm}^{2}$ in our case) and the hole diffusion coefficient in silicon $\left(12 \mathrm{~cm}^{2} / \mathrm{s}\right)$, being distance and time the variables. With these parameters, this theory stays that holes could effectively reach neighboring pixels causing electrical crosstalk.

In order to verify either there exists crosstalk in our devices, a SEM/FIB (a dual-beam Strata 235 from FEI) setup was used to hit one specific sensor with a beam of collimated electrons, $1 \mathrm{~nm}$ in diameter, and to measure the signal generated in its neighbor sensors. Table I summarizes the measurements obtained after hitting on pixel 0 with a beam of electrons accelerated to $1 \mathrm{keV}$. The observation time $\left(\mathrm{t}_{\mathrm{obs}}\right)$, i.e. the time when the sensors are ready for detection, what implies polarization with $\mathrm{V}_{\mathrm{OV}}>0$, was kept as $10 \mathrm{~ns}$. The dead time, in which the ADPs are no more sensitive, was adjusted to make $5 \cdot 10^{6}$ hits in $1 \mathrm{~s}$. The five APDs were polarized only $\mathrm{V}_{\mathrm{OV}}=1.0 \mathrm{~V}$ above their breakdown to minimize the number of hot carriers and so the possibility of optical crosstalk.

Table 1. Counts in dark (DC) and when PIXEL 0 is hit with an electron beam (measurement) for the five pixels in the APD array polarized with $\mathrm{V}_{\mathrm{OV}}=1.0 \mathrm{~V}$. Signal is obtained from the difference between measurement and DC, and $\%$ min and Max are the percentage of (signal-Std.)/(signal in pixel 0) and (signal + Std.)/(signal in pixel 0), when it has sense.

\begin{tabular}{|c|c|c|c|c|c|c|c|c|c|c|}
\hline \multirow{2}{*}{$\mathbf{t}_{\mathbf{o b s}}=10 \mathrm{~ns}$} & \multicolumn{2}{|c|}{ PIXEL 4 } & \multicolumn{2}{c|}{ PIXEL 3 } & \multicolumn{2}{c|}{ PIXEL 2 } & \multicolumn{2}{c|}{ PIXEL 1 } & \multicolumn{2}{c|}{ PIXEL 0 } \\
\cline { 2 - 11 } & Mean & Std. & Mean & Std. & Mean & Std. & Mean & Std. & Mean & Std. \\
\hline DC & 9889 & 89 & 11280 & 109 & 2904 & 47 & 7015 & 80 & 2427 & 56 \\
\hline measurement & 9957 & 99 & 11375 & 82 & 2994 & 76 & 7159 & 90 & 2978 & 63 \\
\hline signal & 68 & 133 & 94 & 136 & 90 & 90 & 143 & 120 & 550 & 59 \\
\hline \% min-Max & - & & - & & - & & 26 & $4-48$ & 100 & \\
\hline
\end{tabular}

Different sensitivity is observed for the different pixels, as indicate the different Dark Count Rates, being pixel 3 the most (11280 counts) and pixel 0 the less (2427 counts) sensitive. This fact influences the total amount of signal (difference between DC and measurement when the electron beam hits pixel 0) detected in each one, giving reason for a fictitious higher value for pixel 3 than for pixel 2. However, signal is smaller or similar to its standard deviation in pixels 4, 3 and 2, debasing its significance. Consequently, only reading in pixel 1 deserves attention, where signal is larger than its deviation, giving a correlation with signal in pixel 0 between 4 and $48 \%$. Error is important, but clearly this signal indicates some influence of the beam hitting in its neighbor pixel. The nature of this influence is not evident, as it can include not only crosstalk but also a tail of the energetic electron beam, which suffers from inelastic dispersion in all directions along the transparent passivation layer and is then collimated but not strictly limited to $1 \mathrm{~nm}$ in diameter. Moreover, exciting with an electron beam such passivated device produces an important charge accumulation that stresses more and more this inelastic dispersion, opening the beam in a larger spot and then influencing more than only one pixel, what prevents long measurements.

All these factors make difficult the measurement with SEM/FIB and their interpretation. Consequently, new approaches for crosstalk characterization were considered as in ${ }^{8,9}$. A new measurement was undertaken in dark at the laboratory, with a sensor chip powered by an Agilent E3631A voltage source and the same control system as before. According to recent improvements in noise thanks to higher repetition times ${ }^{10}$, the crosstalk characterization was performed with an adjustable measurement time that depends on the sensor observation period $\left(\mathrm{t}_{\mathrm{obs}}\right)$ and also on the number of times this observation is repeated $\left(\mathrm{n}_{\text {rep }}\right) . \mathrm{t}_{\text {obs }}$ was adjusted between 2.5 and 40 ns with different overvoltages, and coincidence measurements were performed by evaluating the correlation between output signals generated in dark conditions. 
To quantify the crosstalk probability, a diode with a high dark count rate in a quiet environment is the ideal situation ${ }^{11}$. This diode is considered to be the emitter, which can generate electrical or optical crosstalk to its primary and secondary neighbors. A pulse coincidence between the emitter and one or more of its neighbors within the same active period indicates either a random coincidence of two dark counts or a crosstalk between these diodes. Hence, the timestamp and the value of these two pixels are saved for each repetition of the measurement. The percentage of pulse coincidences for $\mathrm{t}_{\mathrm{obs}}$ of 2.5, 5 and $10 \mathrm{~ns}$ is shown in table 2 . According to table 1, the major sensitivity of pixels 4, 3 and 1 is visible, with large probabilities of coincidences due simply to random simultaneity of dark counts in these pixels. On the other hand, optical crosstalk is also possible, and will be considered later on. Figure 3 summarizes the coincidences in table 2 averaged for the five pixels to minimize the influence of different sensitivities. It seems that observation times around 5 ns are long enough to saturate the cross talk to the values of $2.75 \%$ and $0.25 \%$ for the first and second neighbours, respectively.

Table 2. Percentage of pulse coincidence between an emitter pixel and the other ones in the five APDs array for $t_{\text {obs }}$ of $2.5,5$ and $10 \mathrm{~ns}$ and $\mathrm{V}_{\mathrm{OV}}=1 \mathrm{~V}$ in dark conditions.

\begin{tabular}{|c|c|c|c|c|c|}
\hline & PIX4 & PIX3 & PIX2 & PIX1 & PIX0 \\
\cline { 2 - 6 } $\mathbf{t}_{\text {obs }}$ 2.5 ns & Emitter & 0.34 & 0.01 & 0.07 & 0 \\
\cline { 2 - 6 } & 0.38 & Emitter & 0.01 & 0.02 & 0.01 \\
\cline { 2 - 6 } & 0.18 & 0.18 & Emitter & 0.18 & 0.18 \\
\cline { 2 - 6 } & 0.04 & 0.01 & 0.007 & Emitter & 0.09 \\
\cline { 2 - 6 } & 0 & 0.02 & 0.02 & 0.26 & Emitter \\
\hline \multirow{5}{*}{$\mathbf{t}_{\text {obs }}$ 5 ns } & PIX4 & PIX3 & PIX2 & PIX1 & PIX0 \\
\cline { 2 - 6 } & Emitter & 9.38 & 0.49 & 0.16 & 0.06 \\
\cline { 2 - 6 } & 10.79 & Emitter & 3.09 & 0.87 & 0.09 \\
\cline { 2 - 6 } & 2.75 & 14.86 & Emitter & 30.39 & 2.95 \\
\cline { 2 - 6 } $\mathbf{t}_{\text {obs }}$ 10 ns & 0.09 & 0.40 & 2.87 & Emitter & 5.83 \\
\cline { 2 - 6 } & 0.12 & 0.14 & 1.03 & 21.61 & Emitter \\
\cline { 2 - 6 } & PIX4 & PIX3 & PIX2 & PIX1 & PIX0 \\
\cline { 2 - 6 } & Emitter & 7.63 & 0.48 & 0.02 & 0.06 \\
\cline { 2 - 6 } & 2.98 & Emitter & 2.53 & 0.69 & 0.10 \\
\cline { 2 - 6 } & 0.08 & 0.31 & Emitter & 26.43 & 2.39 \\
\cline { 2 - 6 } & 0.12 & 0.17 & 0.76 & Emitter & 4.50 \\
\hline
\end{tabular}

As it can be seen in table 2 and in figure 3, the dark count rate increases with $\mathrm{t}_{\mathrm{obs}}$ from 2.5 to $5 \mathrm{~ns}$, and therefore random dark count coincidences are more likely. However, the values presented in the table can be taken as the maximum probability of crosstalk. Nevertheless, there is no large difference between 5 and $10 \mathrm{~ns}$, where some kind of saturation is reached. According to the theory described at the beginning of this results section and to ISE-TCAD calculations, the time needed for holes to diffuse until the neighboring pixel (between distances $\mathrm{A}$ and $\mathrm{B}$ in figure 1, depending on the precise point in which the avalanche is generated) is between 0.2 and $6 \mathrm{~ns}$. This should then represent the time necessary to generate crosstalk to the first neighbor. The theoretical results agree with this experiment, demonstrating the possibility of eliminate crosstalk by means of gated operation of the SPAD with $\mathrm{t}_{\mathrm{obs}}$ under $2.5 \mathrm{~ns}$.

Complementarily, optical crosstalk can be produced in several ways. One possible process is due to successive reflections in the transparent passivation layer or even internally off the bottom of the chip, guiding light to a neighbor detector, where it is absorbed generating a new avalanche ${ }^{12}$. Another possibility occurs if the avalanche process produces secondary photons by radiative emission from the hot carriers, which originates spurious avalanches in near detectors ${ }^{13}$. Being the refraction indices of silicon and silicon dioxide of 3.42 and 1.52, respectively, the emission probability about $10^{-5}$ photons per carrier crossing the junction and attenuation length in silicon for near-UV and visible photons around 80 $\mu \mathrm{m}$, the time that light will travel from a detector to its neighbor can be estimated to be of the order of $0.1 \mathrm{ps}$. Optical contribution is then the fast component of crosstalk, and may be minimized by a reduction of the number of hot carriers 
or by means of a suitable optical isolation among diodes. Although, optical crosstalk is usually negligible in monolithic SPAD arrays given the relatively small number of carriers involved in an avalanche in comparison to hybrid devices ${ }^{14}$, in this case can contribute to the small crosstalk observed when short observation times are used.

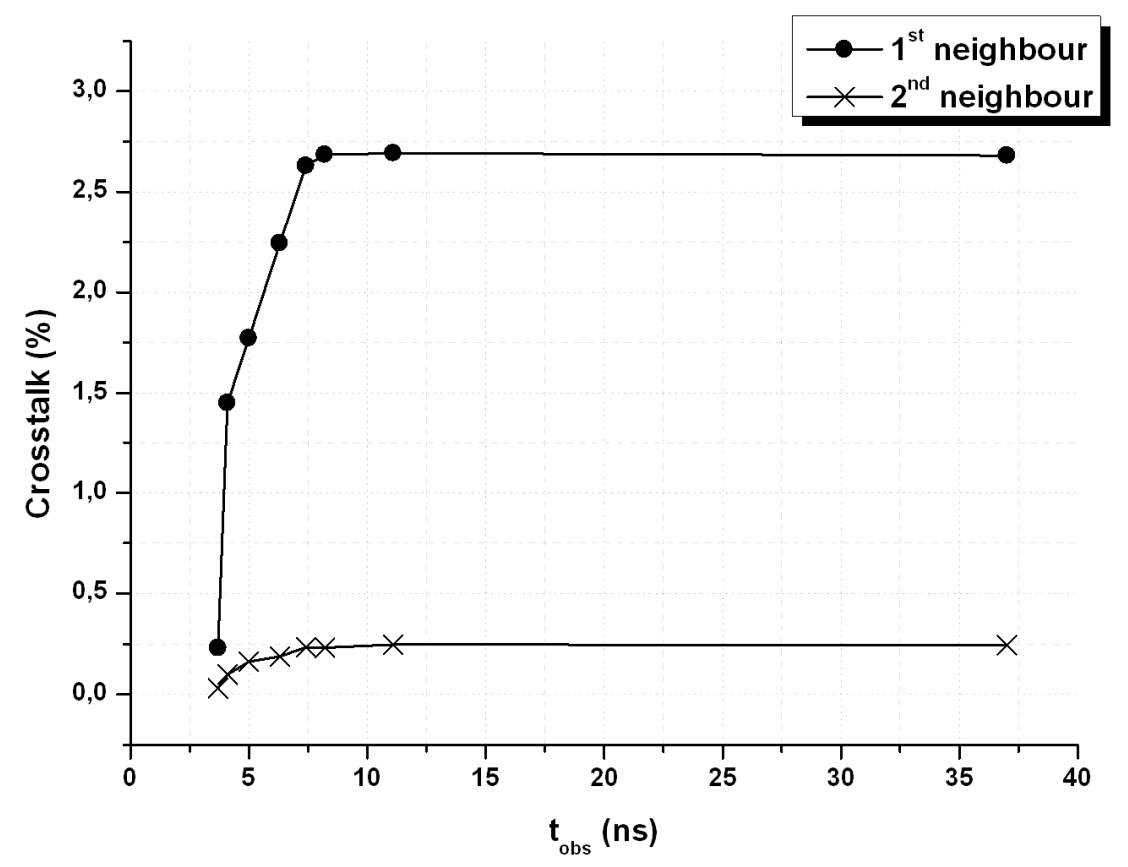

Figure 3. Crosstalk $\%$ for different $\mathrm{t}_{\mathrm{obs}}$ as counted at the laboratory for avalanche coincidences in dark conditions for $\mathrm{V}_{\mathrm{OV}}=$ $1.0 \mathrm{~V}$.

As a result, by using the gated operation with observation times below a few ns allows to reduce the detection of noise and then the minimum detectable light intensity $(\mathrm{SNR}=1)$ decreases also. The upper intensity limit is fix, related to the saturation of the readout electronics. Consequently, the dynamic range (ratio between maximum and minimum detectable intensities) is observed to extend. In many imaging applications this results in a better differentiation between light and darkness, i.e. better contrast. To obtain this quality parameter, the response to a variable optical intensity of a pulsed light should be tested. For the device under test, an IR LED was used in continuous lightning, and two different observation times (14 and $1274 \mathrm{~ns}$ ) were applied $10^{7}$ times to the SPAD. The mean dynamic range of all the pixels was calculated, from the lower LED intensities, where the detected pulses correspond just to noise, to the higher ones, for which the SPAD counts saturate. In these conditions, the dynamic range is found to evolve from the original 9.21 bits to 12.84 bits $^{15}$, providing good spatial resolution and contrast.

\section{CONCLUSIONS}

An array of five $20 \mu \mathrm{m} \times 100 \mu \mathrm{m}$ SPADs has been designed and fabricated in conventional HV-CMOS technology in the same n-well, giving a surface fill factor of $67 \%$. Crosstalk characterization when it is operated in gated mode is reported. Measuring with a long gating time, a crosstalk not less than $2.75 \%$ is observed for the primary neighbor, and $0.25 \%$ for the secondary one. However, it is demonstrated that the reduction of the gating time below $2.5 \mathrm{~ns}$ minimizes the detection of dark counts and eliminates the crosstalk, in good agreement with theory and by TCAD simulations. The technique has shown to provide good spatial resolution and contrast in 2D imaging with the proposed SPAD technology. 


\section{REFERENCES}

[1] Niclass, C., Rochas, A., Besse, P.A., Popovic, R. and Charbon, E., "A $4 \mu$ s integration time imager based on CMOS single photon avalanche diode technology," Sensors \& Actuators A 130-131, 273-281 (2006).

[2] Stoppa, D., Mosconi, D., Panchieri, L. and Gonzo, L., "Single-photon avalanche diode CMOS sensor for timeresolved fluorescence measurements," IEEE Sensors J. 9, 1084-1090 (2009).

[3] Fishburn M.W. and Charbon, E., "System tradeoffs in gamma-ray detection utilizing SPAD arrays and scintillators," IEEE Trans. Nucl. Sci. 57, 2549-2557 (2010).

[4] Vilella E. et al., "Readout electronics for low dark count pixel detectors based on Geiger mode avalanche photodiodes fabricated in conventional CMOS technologies for future linear colliders," Nucl. Instrum. Methods Phys. Res. Sect. A 650, 120-124 (2011).

[5] Niclass, C., Soga, M., Matsubara, H., Kato S. and Kagami, M., "A 100-m range 10-frame/s 340x96-pixel time-toflight depth sensor in 0.18-um CMOS,” IEEE J. Solid-State Cir. 48(2), 559-568 (2013).

[6] Vilella, E., Alonso O. and Dieguez, A., "3D integration of Geiger-mode avalanche photodiodes aimed to very high fill-factor pixels for future linear colliders," to appear in Nucl. Instrum. Methods Phys. Res. Sect. A, 2013. DOI 10.1016/j.nima.2013.05.022.

[7] Vilà, A., Trenado, J., Comerma, A., Gascón, D., Arbat, A., Garrido, L. and Dieguez, A., "FIB-SEM as a tool for characterizing single-photon detectors," Proceedings of the SPIE 7780, 77800Z (2010).

[8] Sciacca, E., Condorelli, G., Aurite, S., Lombardo, S., Mazzillo, M., Sanfilippo, D., Fallica, G. and Rimini, E., "Crosstalk characterization in Geiger-mode avalanche photodiode arrays," IEEE El. Dev. Let. 29(3), 218-220 (2008).

[9] Gerbasch, M., Mayurama, Y., Trimananda, R., Fishburn, M.W., Stoppa, D., Richardson, J.A., Walker, R., Henderson, R. and Charbon, E., "A time-resolved, low-noise single-photon image sensor fabricated in deep-submicron CMOS technology,” IEEE J. Sol. State Cir. 47(6), 1394-1407 (2012).

[10] Mayurama, Y., Blacksberg, J., Rossman, G.R. and Charbon, E., "A time-resolved 128x128 SPAD camera for laser Raman spectroscopy,” Proc. SPIE 8374, 83740N (2012).

[11] Franch, T., Prescher, G., Degenhardt, C., Gruyter, R., Schmitz, A. and Ballizany, R., "The digital photomultiplier Principle of operation and intrinsic detector performance," Proc. IEEE Nucl. Sci. Symp. Conf. Record N28-5, 1959-1965 (2009).

[12] Rech, I., Ingargiola, A., Spinelli, R., Labanca, I., Marangoni, S., Ghioni, M. and Cova, S., "Optical crosstalk in single photon avalanche diode arrays: a now complete model," Optics Express 16 (12), 8381-8394 (2008).

[13] Lacaita, A., Ghioni, M., Cova, S. and Zappa, F., "Ultrafast single-photon avalanche diodes without slow tails in the pulse response,” IEEE Trans. On el. Dev. 40, 2145 (1993).

[14] Niclass, C., Sergio, M. and Charbon, E., "A single photon avalanche diode array fabricated in $0.35 \mu \mathrm{m}$ CMOS and based on an event-driven readout for TCSPC experiments," Proc. SPIE 6372, 63720S (2006).

[15] Vilella, E., Alonso, O., Montiel, A., Vilà, A. and Dieguez, A., "A low-noise time-gated single-photon detector in a HV-CMOS technology for triggered imaging," to appear in Sensors \& Actuators A (2013). 